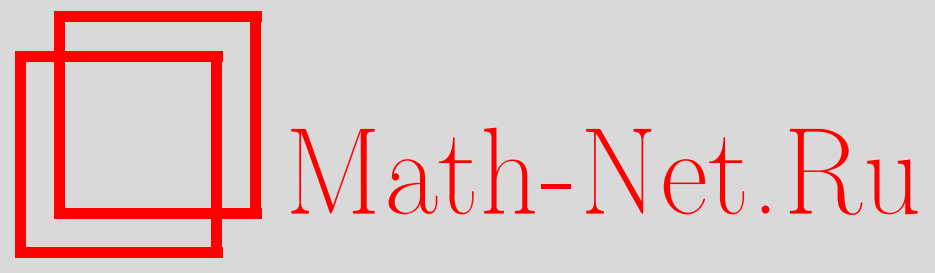

В. Е. Зотеев, Р. Ю. Макаров, Численный метод определения параметров модели ползучести разупрочняющегося материала, Вестн. Сам. гос. техн. ун-та. Сер. Физ.-мат. науки, 2016, номер 2, 328-341

DOI: https://doi.org/10.14498/vsgtu1488

Использование Общероссийского математического портала MathNet.Ru подразумевает, что вы прочитали и согласны с пользовательским соглашением

http: //www . mathnet.ru/rus/agreement

Параметры загрузки:

IP : 52.205 .19 .152

26 апреля 2023 г., 15:41:52

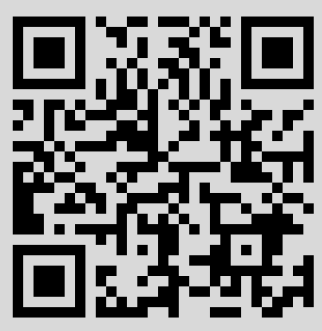


Вестн. Сам. гос. техн. ун-та. Сер. Физ.-мат. науки. 2016. Т. 20, № 2 . С. $328-341$

ISSN: 2310-7081 (online), 1991-8615 (print)

doi: http://dx.doi.org/10.14498/vsgtu1488

Математическое моделирование,
численные методы и комплексы
программ

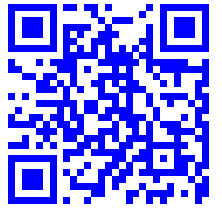

УДК 519.651.3:539.376

\title{
ЧИСЛЕННЫЙ МЕТОД ОПРЕДЕЛЕНИЯ ПАРАМЕТРОВ МОДЕЛИ ПОЛЗУЧЕСТИ РАЗУПРОЧНЯЮЩЕГОСЯ МАТЕРИАЛА
}

\author{
В. Е. Зотеев, Р. Ю. Макаров \\ Самарский государственный технический университет, \\ Россия, 443100, Самара, ул. Молодогвардейская, 244.
}

\begin{abstract}
Аннотация
Тенденции к уменьшению массы машин при улучшении их качества, а также стремление к наиболее полному использованию механических свойств материалов требуют постоянного совершенствования и развития известных методов расчета и анализа напряженно-деформированного состояния материалов в условиях ползучести. В статье рассматривается новый численный метод оценки параметров математической модели ползучести разупрочняющегося материала на основе экспериментальных диаграмм, построенных по результатам испытаний при различных напряжениях. В основе метода лежит обобщенная регрессионная модель, построенная на основе разностных уравнений, описывающих диаграммы ползучести. Полученные соотношения между коэффициентами разностного уравнения и параметрами деформации ползучести позволяют свести задачу параметрической идентификации к итерационной процедуре среднеквадратичного оценивания коэффициентов, линейной на каждом шаге итерации обобщенной регрессионной модели. Проведена апробация разработанного численного метода на пяти экспериментальных кривых ползучести алюминиевого сплава, подтверждающая достоверность полученных соотношений и эффективность численного метода.
\end{abstract}

Ключевые слова: ползучесть разупрочняющегося материала, разностные уравнения, обобщенная регрессионная модель, нелинейная регрессия, среднеквадратическое оценивание.

(C) 2016 Самарский государственный технический университет.

\section{Образец для цитирования}

Зотеев В. Е., Макаров Р. Ю. Численный метод определения параметров модели ползучести разупрочняющегося материала // Вестн. Сам. гос. техн. ун-та. Сер. Физ.-мат. науки, 2016. Т. 20, № 2. C. 328-341. doi: 10.14498/vsgtu1488.

\section{Сведения об авторах}

Владимир Евгенъевич Зотеев (д.т.н., доц.; zoteev-ve@mail.ru; автор, ведущий переписку), профессор, каф. прикладной математики и информатики.

Роман Юръевич Макаров (makaroman1@yandex.ru), аспирант, каф. прикладной математики и информатики. 
Введение. Исследование поведения материалов в условиях ползучести является важной научно-технической задачей. Тенденции к уменьшению массы машин при улучшении их качества, а также стремление к наиболее полному использованию механических свойств материалов требуют совершенных методов расчета, в которых наиболее полно отражены механические свойства материалов [1]. Однако нелинейность определяющих уравнений ползучести и длительной прочности существенно затрудняет применение аналитических и повышает роль численных методов решения.

При высоких температурах характерными особенностями деформационно-прочностного поведения материалов являются отсутствие начальной стадии ползучести (стадия упрочнения) и сравнительно небольшая продолжительность третьей стадии (стадия разупрочнения) [2]. Вместе с тем именно третья стадия ползучести как стадия, предшествующая разрушению, является определяющей при расчете срока службы проектируемого элемента конструкции по катастрофическому критерию отказа [3]. Большинство существующих методик определения параметров моделей ползучести разупрочняющихся материалов обладают одним существенным недостатком. Они не обеспечивают достаточную адекватность построенной модели данным эксперимента, вследствие чего обладают низкой помехозащищенностью и точностью оценок параметров модели [3-5]. Для устранения этого недостатка в данной работе предлагается новый численный метод нелинейного оценивания, в основе которого лежат разностные уравнения, описывающие диаграммы ползучести материала при различных постоянных номинальных напряжениях.

Определяющие уравнения. При математическом моделировании процессов деформирования и разрушения материалов используем энергетический подход, предложенный в [5]. Этот подход базируется на принципе суперпозиции упругой, пластической деформаций и деформации ползучести, а также методе разделения деформации ползучести. Основной вариант определяющих соотношений представлен в [5]. Из этих соотношений с учетом отсутствия вязкоупругой и вязкопластической составляющих в деформации ползучести $p$ вытекают уравнения

$$
\dot{p}=c \sigma^{m}, \quad \sigma=\sigma_{0}(1+\omega), \quad \dot{\omega}=\alpha \sigma \dot{p},
$$

где $\sigma_{0}$ и $\sigma$-соответственно номинальное и истинное напряжения $\left(\sigma_{0} \geqslant 0\right)$; $\omega$ - параметр поврежденности; $c$ и $m$ - константы модели, при помощи которых описывается вторая стадия ползучести; $\alpha$ - параметр модели, контролирующий процесс разупрочнения материала на третьей стадии ползучести. Из этой системы дифференциальных уравнений для нулевых начальных условий можно построить временные зависимости деформации ползучести

$$
p\left(t, \sigma_{0 j}\right)=-\frac{1}{\sigma_{0 j} m \alpha} \ln \left(1-\alpha m c \sigma_{0 j}^{m+1} t\right),
$$

которые могут быть использованы для описания третьей стадии ползучести при различных постоянных значениях номинального напряжения $\sigma_{0 j}$. Очевидно, что для третьей стадии ползучести важнейшим участком моделирования является промежуток времени непосредственно перед моментом разрушения материала, когда кривая ползучести практически совпадает с вертикальной асимптотой. Поэтому при выборе критерия «близости» модели 
к эксперименту необходимо в первую очередь ориентироваться на величину отклонения прогнозируемого времени разрушения от данных эксперимента. Это означает, что при оценке параметров математической модели (1) следует минимизировать норму разности по временной координате:

$$
\|\varepsilon\|^{2}=\|t-\hat{t}\|^{2} \rightarrow \min
$$

где $t-\left(\sum_{j=1}^{M} N_{j}\right)$-мерный вектор данных эксперимента $t_{k, j}, k=0,1,2, \ldots$, $N_{j}-1$, вычисленный по $M$ диаграммам ползучести, в каждой из которых взято по $N_{j}$ точек, $j=1,2, \ldots, M ; \hat{t}-\left(\sum_{j=1}^{M} N_{j}\right)$-мерный вектор результатов вычислений $\hat{t}_{k, j}, k=0,1,2, \ldots, N_{j}-1$, по модели (1) при значениях деформации ползучести $p_{k, j}$ в соответствующих точкам эксперимента $\left(t_{k, j}, p_{k, j}\right), k=$ $=0,1,2, \ldots, N_{j}-1, j=1,2, \ldots, M$. При этом зависимость $\hat{t}_{k, j}$ от $p_{k, j}$ в явном виде можно получить из выражения (1):

$$
\begin{gathered}
\hat{t}_{k, j}=\frac{1}{c m \sigma_{0 j}^{m+1} \alpha}\left[1-\exp \left(-m \alpha \sigma_{0 j} p_{k, j}\right)\right], \\
k=0,1,2, \ldots, N_{j}-1, \quad j=1,2, \ldots, M .
\end{gathered}
$$

Таким образом, задача параметрической идентификации модели для описания третьей стадии ползучести сводится к минимизации нелинейной функции от трех переменных $c, m$ и $\alpha$ :

$$
J=\sum_{j=1}^{M} \sum_{k=0}^{N_{j}-1}\left\{t_{k, j}-\frac{1}{c m \sigma_{0 j}^{m+1} \alpha}\left[1-\exp \left(-m \alpha \sigma_{0 j} p_{k, j}\right)\right]\right\}^{2} \rightarrow \min .
$$

Данная задача относится к классу задач нелинейной регрессии, методы исследования которой описаны в [6] и сопряжены со сложностью поиска решения нормальной системы нелинейных уравнений. В данной работе предлагается новый численный метод решения задачи нелинейной регрессии, в основе которого лежат разностные уравнения, описывающие результаты эксперимента. Такой подход позволяет свести решение нелинейной задачи к итерационной процедуре, на каждом шаге которой оценки коэффициентов разностного уравнения вычисляются на основе линейной обобщенной регрессионной модели [7-10]. Численный метод оценки параметров рассматриваемой модели ползучести на основе разностных уравнений по совокупности $M$ экспериментально построенных при $\sigma_{0 j}=$ const кривых включает следующие шаги:

- предварительная обработка выборки результатов наблюдений для каждой $j$-той кривой с целью равномерной дискретизации данных эксперимента по переменной $p_{k, j}=\Delta p_{j} k$, где $\Delta p_{j}$-шаг (период) дискретизации;

- построение разностных уравнений, связывающих несколько последовательных значений дискретной модели (2), коэффициенты которых известным образом связаны с параметрами $c, m$ и $\alpha$ модели (1);

- формирование обобщенной регрессионной модели, описывающей результаты эксперимента и линейной относительно коэффициентов разностного уравнения; 
- итерационная процедура среднеквадратичного оценивания коэффициентов обобщенной регрессионной модели;

- вычисление оценок параметров деформации ползучести на основе среднеквадратичных оценок коэффициентов обобщенной регрессионной модели;

- статистическая оценка погрешности результатов вычисления и построение доверительных интервалов для каждой из $M$ кривых ползучести.

Построение разностных уравнений и обобщенной регрессионной модели, описывающих результаты эксперимента. На первом шаге численного метода проводится предварительная обработка результатов эксперимента для каждой из $M$ кривых ползучести, построенных при постоянных значениях номинального напряжения $\sigma_{0 j}=$ const. Предварительная обработка заключается в сглаживании результатов наблюдений с помощью скользящих средних и формировании равномерной (по переменной $p_{k, j}$ с шагом $\Delta p_{j}$ ) выборки экспериментальных данных заданного объема $N_{j}$. Как показали численно-аналитические исследования процедуры предварительной обработки, систематическая погрешность от сглаживания на основе скользящих средних в точках $\left(p_{k, j}, t_{k, j}\right)$ эксперимента на два порядка меньше, чем величина $\varepsilon_{k, j}$ естественного разброса результатов наблюдений.

В основе построения разностных уравнений, связывающих несколько последовательных значений дискретной модели

$$
\begin{gathered}
\hat{t}_{k, j}=\frac{1}{c m \sigma_{0 j}^{m+1} \alpha}\left[1-\exp \left(-m \alpha \sigma_{0 j} \Delta p_{j} k\right)\right], \\
k=0,1,2, \ldots, N_{j}-1, \quad j=1,2, \ldots, M
\end{gathered}
$$

лежит методика, описанная в [7]. Из формулы (3) при $k=2,3, \ldots, N_{j}, j=$ $=1,2, \ldots, M$ имеем следующее:

$$
c m \sigma_{0 j}^{m+1} \alpha \hat{t}_{k, j}=1-\exp \left(-m \alpha \sigma_{0 j} \Delta p_{j} k\right),
$$

$$
\begin{aligned}
& c m \sigma_{0 j}^{m+1} \alpha \hat{t}_{k-1, j}=1-\exp \left[-m \alpha \sigma_{0 j} \Delta p_{j}(k-1)\right]= \\
& =1-\exp \left(-m \alpha \sigma_{0 j} \Delta p_{j} k\right) \exp \left(m \alpha \sigma_{0 j} \Delta p_{j}\right),
\end{aligned}
$$

$$
\begin{aligned}
& c m \sigma_{0 j}^{m+1} \alpha \hat{t}_{k-2, j}=1-\exp \left[-m \alpha \sigma_{0 j} \Delta p_{j}(k-2)\right]= \\
& \quad=1-\exp \left(-m \alpha \sigma_{0 j} \Delta p_{j} k\right) \exp \left(2 m \alpha \sigma_{0 j} \Delta p_{j}\right) .
\end{aligned}
$$

Отсюда, переходя к разностям, можно получить соотношение

$$
\ln \frac{\hat{t}_{k-1, j}-\hat{t}_{k-2, j}}{\hat{t}_{k, j}-\hat{t}_{k-1, j}}=\sigma_{0 j} \Delta p_{j} \lambda_{1}, \quad k=2,3, \ldots, N_{j}, j=1,2, \ldots, M,
$$

где $\lambda_{1}=m \alpha$.

При $k=0$ и $k=1$ из формулы $(3)$, соответственно, получаем

$$
\hat{t}_{0, j}=0, \quad j=1,2, \ldots, M
$$


И

$$
\hat{t}_{1, j}=\frac{1}{c m \sigma_{0 j}^{m+1} \alpha}\left[1-\exp \left(-m \alpha \sigma_{0 j} \Delta p_{j}\right)\right], \quad j=1,2, \ldots, M .
$$

Из последнего соотношения имеем

$$
\exp \left(\lambda_{1} \sigma_{0 j} \Delta p_{j}\right) c m \alpha \sigma_{0 j}^{m+1} \hat{t}_{1, j}=\exp \left(\lambda_{1} \sigma_{0 j} \Delta p_{j}\right)-1
$$

Отсюда после логарифмирования получаем

$$
\ln \left[\exp \left(\lambda_{1} \sigma_{0 j} \Delta p_{j}\right)-1\right]-\ln \hat{t}_{1, j}=\lambda_{1} \sigma_{0 j} \Delta p_{j}+\lambda_{2}+\lambda_{3} \ln \sigma_{0 j}, \quad j=1,2, \ldots, M,
$$

где $\lambda_{2}=\ln (c m \alpha)$ и $\lambda_{3}=m+1$.

Таким образом, имеем систему разностных уравнений, связывающих последовательность $\hat{t}_{k-2, j}, \hat{t}_{k-1, j}$ и $\hat{t}_{k, j}$ дискретных значений модели $(3), j=$ $=1,2, \ldots, M$ :

$$
\left\{\begin{array}{l}
\hat{t}_{0, j}=0 \\
\ln \left[\exp \left(\lambda_{1} \sigma_{0 j} \Delta p_{j}\right)-1\right]-\ln \hat{t}_{1, j}=\lambda_{1} \sigma_{0 j} \Delta p_{j}+\lambda_{2}+\lambda_{3} \ln \sigma_{0 j} \\
\ln \frac{\hat{t}_{k-1, j}-\hat{t}_{k-2, j}}{\hat{t}_{k, j}-\hat{t}_{k-1, j}}=\sigma_{0 j} \Delta p_{j} \lambda_{1}, \quad k=2,3, \ldots, N_{j}-1 .
\end{array}\right.
$$

Коэффициенты в системе разностных уравнений (4) связаны с параметрами деформации ползучести соотношениями

$$
\lambda_{1}=m \alpha, \quad \lambda_{2}=\ln (c m \alpha), \quad \lambda_{3}=m+1,
$$

которые позволяют по оценкам коэффициентов $\lambda_{j}$ вычислять оценки параметров деформации ползучести.

Используем соотношения

$$
t_{k, j}=\hat{t}_{k, j}+\varepsilon_{k, j}, \quad k=0,1,2, \ldots, N_{j}-1, j=1,2, \ldots, M,
$$

где $t_{k, j}$ - результаты эксперимента, т.е. значения переменной $t$ при аргументе $\Delta p_{j} k$ для каждой $j$-той кривой; $\varepsilon_{k, j}$ - естественный разброс результатов наблюдения относительно модели (3). После линеаризации по переменным $\varepsilon_{1, j}$, $\varepsilon_{k-2, j}, \varepsilon_{k-1, j}$ и $\varepsilon_{k, j}$, в первом приближении получаем

$$
\left\{\begin{array}{l}
\ln \hat{t}_{1, j}=\ln \left(t_{1, j}-\varepsilon_{1, j}\right) \approx \ln t_{1, j}-\frac{\varepsilon_{1, j}}{t_{1, j}} \\
\ln \frac{\hat{t}_{k-1, j}-\hat{t}_{k-2, j}}{\hat{t}_{k, j}-\hat{t}_{k-1, j}}=\ln \frac{t_{k-1, j}-t_{k-2, j}-\left(\varepsilon_{k-1, j}-\varepsilon_{k-2, j}\right)}{t_{k, j}-t_{k-1, j}-\left(\varepsilon_{k, j}-\varepsilon_{k-1, j}\right)} \approx \\
\approx \ln \frac{t_{k-1, j}-t_{k-2, j}}{t_{k, j}-t_{k-1, j}}+\frac{1}{t_{k-1, j}-t_{k-2, j}} \varepsilon_{k-2, j}- \\
-\frac{t_{k, j}-t_{k-2, j}}{\left(t_{k-1, j}-t_{k-2, j}\right)\left(t_{k, j}-t_{k-1, j}\right)} \varepsilon_{k-1, j}+\frac{1}{t_{k, j}-t_{k-1, j}} \varepsilon_{k, j} .
\end{array}\right.
$$

Подставляя формулы (5) в систему уравнений (4), имеем математическую модель, которая в форме разностных уравнений описывает результаты эксперимента $\left(p_{k, j}, t_{k, j}\right), k=0,1,2, \ldots, N_{j}-1, j=1,2, \ldots, M$. 
Полагая $\varepsilon_{0, j}=0$ с учетом нулевых начальных условий

$$
p_{0, j}=0, \quad t_{0, j}=0,
$$

получаем

$$
\left\{\begin{array}{l}
t_{0, j}=0 ; \\
\ln \frac{\exp \left(\lambda_{1} \sigma_{0 j} \Delta p_{j}\right)-1}{t_{1, j}}=\lambda_{1} \sigma_{0 j} \Delta p_{j}+\lambda_{2}+\lambda_{3} \ln \sigma_{0 j}-\frac{\varepsilon_{1, j}}{t_{1, j}} \\
\ln \frac{t_{k-1, j}-t_{k-2, j}}{t_{k, j}-t_{k-1, j}}=\sigma_{0 j} \Delta p_{j} \lambda_{1}-\frac{1}{t_{k-1, j}-t_{k-2, j}} \varepsilon_{k-2, j}+ \\
\quad+\frac{t_{k, j}-t_{k-2, j}}{\left(t_{k-1, j}-t_{k-2, j}\right)\left(t_{k, j}-t_{k-1, j}\right)} \varepsilon_{k-1, j}-\frac{1}{t_{k, j}-t_{k-1, j}} \varepsilon_{k, j}
\end{array}\right.
$$

где $k=2,3, \ldots, N_{j}-1, j=1,2, \ldots, M$.

Система разностных уравнений (6) лежит в основе формирования обобщенной регрессионной модели. Введем следующие векторы и матрицы:

$-\lambda=\left[\begin{array}{lll}\lambda_{1} & \lambda_{2} & \lambda_{3}\end{array}\right]^{\top}$ - трехмерный вектор коэффициентов обобщенной регрессионной модели;

$-b_{\lambda}=\left[\begin{array}{llll}b_{1} & b_{2} & \cdots & b_{M}\end{array}\right]^{\top}$ - блочный вектор размера $\left(\sum_{j=1}^{M} N_{j} \times 1\right)$, состоящий из $M$ векторов $b_{j}$ размера $\left(N_{j} \times 1\right)$ вида

$$
b_{j}=\left[\begin{array}{c}
t_{0, j} \\
\ln \frac{\exp \left(\lambda_{1} \sigma_{0 j} \Delta p_{j}\right)-1}{t_{1, j}} \\
\ln \frac{t_{1, j}-t_{0, j}}{t_{2, j}-t_{1, j}} \\
\ln \frac{t_{2, j}-t_{1, j}}{t_{3, j}-t_{2, j}} \\
\vdots \\
\ln \frac{t_{N_{j}-2, j}-t_{N_{j}-3, j}}{t_{N_{j}-1, j}-t_{N_{j}-2, j}}
\end{array}\right], \quad j=1,2, \ldots, M
$$

- $F=\left[\begin{array}{llll}F_{1} & F_{2} & \cdots & F_{M}\end{array}\right]^{\top}$ - блочная матрица размера $\left(\sum_{j=1}^{M} N_{j} \times 3\right)$, состоящая из $M$ матриц $F_{j}$ размера $\left(N_{j} \times 3\right)$ вида

$$
F_{j}=\left[\begin{array}{ccc}
0 & 0 & 0 \\
\sigma_{0 j} \Delta p_{j} & 1 & \ln \sigma_{0 j} \\
\sigma_{0 j} \Delta p_{j} & 0 & 0 \\
\vdots & \vdots & \vdots \\
\sigma_{0 j} \Delta p_{j} & 0 & 0
\end{array}\right], \quad j=1,2, \ldots, M
$$

- $\varepsilon=\left[\begin{array}{llll}\varepsilon_{1}^{\top} & \varepsilon_{2}^{\top} & \cdots & \varepsilon_{M}^{\top}\end{array}\right]^{\top}$ - блочный вектор естественного разброса данных эксперимента относительно модели (остатков) размера $\left(\sum_{j=1}^{M} N_{j} \times 1\right)$, состоящий из $M$ векторов $\varepsilon_{j}$ размера $\left(N_{j} \times 1\right)$ :

$$
\varepsilon_{j}=\left[\begin{array}{lllll}
\varepsilon_{0, j} & \varepsilon_{1, j} & \varepsilon_{2, j} & \cdots & \varepsilon_{N-1, j}
\end{array}\right]^{\top}, \quad j=1,2, \ldots, M
$$


- $\eta=\left[\begin{array}{llll}\eta_{1}^{\top} & \eta_{2}^{\top} & \cdots & \eta_{M}^{\top}\end{array}\right]^{T}$ - блочный вектор «невязки» обобщенной регрессионной модели размера $\left(\sum_{j=1}^{M} N_{j} \times 1\right)$, состоящий из $M$ векторов $\eta_{j}$ размера $\left(N_{j} \times 1\right)$ :

$$
\eta_{j}=\left[\begin{array}{lllll}
\eta_{1, j} & \eta_{2, j} & \eta_{3, j} & \cdots & \eta_{N, j}
\end{array}\right]^{\top}, \quad j=1,2, \ldots, M,
$$

где

$$
\begin{gathered}
\eta_{1, j}=\varepsilon_{0, j}=0, \quad \eta_{2, j}=-\frac{\varepsilon_{1, j}}{t_{1, j}} \\
\eta_{i, j}=-\frac{1}{t_{i-2, j}-t_{i-3, j}} \varepsilon_{i-3, j}+\frac{t_{i-1, j}-t_{i-3, j}}{\left(t_{i-2, j}-t_{i-3, j}\right)\left(t_{i-1, j}-t_{i-2, j}\right)} \varepsilon_{i-2, j}- \\
-\frac{1}{t_{i-1, j}-t_{i-2, j}} \varepsilon_{i-1, j}, \quad i=3,4, \ldots, N_{j} ;
\end{gathered}
$$

$-P=\left[\begin{array}{ccccc}P_{1} & \Theta & \Theta & \ldots & \Theta \\ \Theta & P_{2} & \Theta & \ldots & \Theta \\ \Theta & \Theta & P_{3} & \ldots & \Theta \\ \vdots & \vdots & \vdots & \ddots & \vdots \\ \Theta & \Theta & \Theta & \ldots & P_{M}\end{array}\right]$ - блочно-диагональная квадратная матрица линейного преобразования вектора остатков размера $\left(\sum_{j=1}^{M} N_{j} \times\right.$ $\left.\times \sum_{j=1}^{M} N_{j}\right)$, состоящая из $M$ матриц $P_{j}$ размера $\left(N_{j} \times N_{j}\right), j=1,2, \ldots$, $M$, и нулевых матриц $\Theta$. Квадратные матрицы $P_{j}$ - нижние треугольные трехдиагональные матрицы, элементы которых описываются следующим образом:

- диагональные элементы:

$$
p_{i, i}^{j}=\left\{\begin{aligned}
1, & i & =1 \\
-\frac{1}{t_{1, j}}, & & i=2 \\
-\frac{1}{t_{i-1, j}-t_{i-2, j}}, & & i=3,4, \ldots, N_{j}
\end{aligned}\right.
$$

- поддиагональные элементы:

$$
p_{i, i-1}^{j}=\left\{\begin{array}{cl}
0, & i=2 ; \\
\frac{t_{i-1, j}-t_{i-3, j}}{\left(t_{i-2, j}-t_{i-3, j}\right)\left(t_{i-1, j}-t_{i-2, j}\right)}, & i=3,4, \ldots, N_{j} ;
\end{array}\right.
$$

- подподдиагональные элементы:

$$
p_{i, i-2}^{j}=-\frac{1}{t_{i-2, j}-t_{i-3, j}}, \quad i=3,4, \ldots, N_{j} .
$$

Остальные элементы матриц $P_{j}$ равны нулю: $p_{k, i}^{j}=0$ при $i>k$ и $i<k-2, k, i=1,2, \ldots, N_{j}$. 
Таким образом, с учетом введенных выше векторов и матриц получаем обобщенную регрессионную модель в виде

$$
\left\{\begin{array}{l}
b_{\lambda}=F \lambda+\eta \\
\eta=P \varepsilon
\end{array}\right.
$$

\section{Итерационная процедура среднеквадратического оценивания коэффици-} ентов обобщенной регрессионной модели. В соответствие с выбранным критерием «близости» математической модели (3) экспериментальным данным $\left(p_{k, j}, t_{k, j}\right)$ минимизируется остаточная сумма квадратов

$$
Q=\|\varepsilon\|^{2}=\|t-\hat{t}\|^{2} \rightarrow \min ,
$$

которую с учетом системы (12) при невырожденной матрице $P$ можно представить в виде

$$
Q=\|\varepsilon\|^{2}=\left\|P^{-1} b_{\lambda}-P^{-1} F \lambda\right\|^{2}
$$

или

$$
Q=\left(b_{\lambda}-F \lambda\right)^{\top} \Omega^{-1}\left(b_{\lambda}-F \lambda\right)=b_{\lambda}^{\top} \Omega^{-1} b_{\lambda}-2 b_{\lambda}^{\top} \Omega^{-1} F \lambda+\lambda^{\top} F^{\top} \Omega^{-1} F \lambda,
$$

где $\Omega=P P^{\top}$ - матрица размера $\left(\sum_{j=1}^{M} N_{j} \times \sum_{j=1}^{M} N_{j}\right)$. Дифференцируя остаточную сумму квадратов по переменным $\lambda_{i}$ и приравнивая нулю результаты дифференцирования, получаем систему нормальных уравнений, которая в векторной форме имеет вид

$$
\left(F-W_{\lambda}\right)^{\top} \Omega^{-1} F \lambda=\left(F-W_{\lambda}\right)^{\top} \Omega^{-1} b_{\lambda},
$$

где блочная матрица $W_{\lambda}=\left[\begin{array}{llll}W_{1} & W_{2} & \cdots & W_{M}\end{array}\right]^{\top}$ размера $\left(\sum_{j=1}^{M} N_{j} \times 3\right)$, состоящая из $M$ матриц размера $\left(N_{j} \times 3\right)$ вида

$$
W_{j}=\left[\begin{array}{ccc}
0 & 0 & 0 \\
\frac{\sigma_{0 j} \Delta p_{j}}{1-\exp \left(-\lambda_{1} \sigma_{0 j} \Delta p_{j}\right)} & 0 & 0 \\
0 & 0 & 0 \\
\vdots & \vdots & \vdots \\
0 & 0 & 0
\end{array}\right], \quad j=1,2, \ldots, M
$$

Так как элементы

$$
w_{l_{j}, 1}=\frac{\sigma_{0 j} \Delta p_{j}}{1-\exp \left(-\lambda_{1} \sigma_{0 j} \Delta p_{j}\right)}
$$

матрицы $W_{\lambda}$ и элементы

$$
b_{l_{j}}=\ln \frac{\exp \left(\lambda_{1} \sigma_{0 j} \Delta p_{j}\right)-1}{t_{1, j}}
$$


вектора $b_{\lambda}$, где $l_{j}=2+\sum_{k=1}^{j-1} N_{k}, j=1,2, \ldots, M, l_{1}=2$, зависят от величины $\lambda_{1}$, система уравнений (13), вообе говоря, нелинейная. Однако она легко разрешается относительно вектора коэффициентов $\lambda$ :

$$
\lambda=\left[\left(F-W_{\lambda}\right)^{\top} \Omega^{-1} F\right]^{-1}\left(F-W_{\lambda}\right)^{\top} \Omega^{-1} b_{\lambda} .
$$

Формула (15) лежит в основе итерационной процедуры среднеквадратичного оценивания элементов вектора $\lambda$. Рекуррентное соотношение, описывающее итерационный процесс на основе (15), имеет вид

$$
\hat{\lambda}^{(i+1)}=\left[\left(F-W_{\hat{\lambda}}^{(i)}\right)^{\top} \Omega^{-1} F\right]^{-1}\left(F-W_{\hat{\lambda}}^{(i)}\right)^{\top} \Omega^{-1} b_{\hat{\lambda}}^{(i)}, \quad i=0,1,2,3, \ldots,
$$

где матрица $W_{b}^{(i)}$ и вектор $b_{\lambda}^{(i)}$ на $i$-том шаге вычисляются на основе $i$-того приближения $\hat{\lambda}_{1}^{(i)}$.

В соответствии с моделью (6) оценку начального приближения $\hat{\lambda}_{1}^{(0)}$ для первого элемента вектора $\lambda=\left[\begin{array}{lll}\lambda_{1} & \lambda_{2} & \lambda_{3}\end{array}\right]^{\top}$ можно найти из условия минимизации

$$
\sum_{j=1}^{M} \sum_{k=2}^{N_{j}-1}\left[\ln \frac{t_{k-1, j}-t_{k-2, j}}{t_{k, j}-t_{k-1, j}}-\sigma_{0 j} \Delta p_{j} \lambda_{1}^{(0)}\right]^{2} \rightarrow \min
$$

Отсюда получаем следующую оценку:

$$
\hat{\lambda}_{1}^{(0)}=\frac{\sum_{j=1}^{M} \sum_{k=2}^{N_{j}-1} \sigma_{0 j} \Delta p_{j} \ln \frac{t_{k-1, j}-t_{k-2, j}}{t_{k, j}-t_{k-1, j}}}{\sum_{j=1}^{M}\left(N_{j}-2\right) \sigma_{0 j}^{2} \Delta p_{j}^{2}} .
$$

Алгоритм итерационной процедуры среднеквадратичного оценивания коэффициентов обобщенной регрессионной модели может быть описан следующим образом.

1. По формулам (8) и (9)-(11) вычисляются элементы матрицы регрессоров $F$ и матрицы линейного преобразования вектора остатков $P$ соответственно.

2. По формуле (17) вычисляется начальное приближение $\hat{\lambda}_{1}^{(0)}$ первой компоненты вектора коэффициентов $\lambda$ системы разностных уравнений (6).

3. По формулам (7) и (14) при значении $\lambda_{1}=\hat{\lambda}_{1}^{(i)}$ при $i=0$ вычисляются элементы вектора $b_{\lambda}$ и матрицы производных $W_{\lambda}$.

4. Вычисляются правая и левая части системы нормальных уравнений (13).

5. По формуле (16) находится приближение $\hat{\lambda}^{(i+1)}$ вектора коэффициентов обобщенной регрессионной модели (12).

6. Сравниваются по модулю величины $\hat{\lambda}_{1}^{(i+1)}$ и $\hat{\lambda}_{1}^{(i)}$. Если $\left|\hat{\lambda}_{1}^{(i+1)}-\hat{\lambda}_{1}^{(i)}\right|<\delta$, где $\delta>0$ - заданное значение предельной абсолютной погрешности, то процесс вычислений заканчивается и за вектор оценок коэффициентов обобщенной регрессионной модели принимается вектор $\hat{\lambda}^{(i+1)}$. Если $\left|\hat{\lambda}_{1}^{(i+1)}-\hat{\lambda}_{1}^{(i)}\right| \geqslant \delta$, то, увеличив $i$ на единицу, следует вернуться к шагу 3 данного алгоритма вычислений. 
По найденным оценкам коэффициентов системы разностных уравнений (6) оценки параметров модели (1) можно вычислить по формулам

$$
\hat{c}=\frac{\exp \hat{\lambda}_{2}}{\hat{\lambda}_{1}}, \quad \hat{m}=\hat{\lambda}_{3}-1, \quad \hat{\alpha}=\frac{\hat{\lambda}_{1}}{\hat{\lambda}_{3}-1} .
$$

\section{Апробация численного метода оценки параметров модели ползучести разу-} прочняющегося материала на основе разностных уравнений. Описанный выше алгоритм численного метода оценки параметров модели (1) был апробирован по данным работы [11] при обработке пяти экспериментальных кривых ползучести алюминиевого сплава Д16Т (пруток диаметром 40 мм) при температуре $250^{\circ} \mathrm{C}$ при испытаниях на растяжение и сжатие (при постоянных номинальных напряжениях $\sigma_{0}=98.1,88.3,78.5,73.6$ и 68.6 МПа.

На этапе предварительной обработки экспериментальных данных были сформированы равномерные по координате $p$ выборки равного объема $N_{j}=20$, $j=1,2,3,4,5$ со следующими периодами дискретизации: $\Delta p_{1}=0.00522$, $\Delta p_{2}=0.00572, \Delta p_{3}=0.00647, \Delta p_{4}=0.00699$ и $\Delta p_{5}=0.00633$. При этом установлено, что предварительная обработка результатов эксперимента на основе сглаживания с помощью скользящего среднего практически не меняет исходные диаграммы ползучести.

В процессе реализации описанного выше алгоритма численного метода оценки параметров модели (1) получены следующие промежуточные результаты.

Начальное приближение $\lambda_{1}^{(0)}$ первой компоненты вектора коэффициентов системы разностных уравнений, вычисленное по формуле (17), следующее:

$$
\hat{\lambda}_{1}^{(0)}=\frac{\sum_{j=1}^{5} \sum_{k=2}^{19} \sigma_{0 j} \Delta p_{j} \ln \frac{t_{k-1, j}-t_{k-2, j}}{t_{k, j}-t_{k-1, j}}}{18 \sum_{j=1}^{5} \sigma_{0 j}^{2} \Delta p_{j}^{2}}=0.6471 .
$$

В соответствии с алгоритмом была реализована итерационная процедура среднеквадратичного оценивания коэффициентов обобщенной регрессионной модели, в результате после 5 итераций были получены коэффициенты обобщенной регрессионной модели:

$$
\hat{\lambda}_{1}=0.861, \quad \hat{\lambda}_{2}=-29.708, \quad \hat{\lambda}_{3}=5.4449 \text {. }
$$

Отсюда, используя соотношения (18), находим оценки параметров модели $(1)$ :

$$
\hat{c}=\frac{\exp \left(\hat{\lambda}_{2}\right)}{\hat{\lambda}_{1}}=1.46 \cdot 10^{-13}, \quad \hat{m}=\hat{\lambda}_{3}-1=4.449, \quad \hat{\alpha}=\frac{\hat{\lambda}_{1}}{\hat{\lambda}_{3}-1}=0.192 .
$$

Таким образом, построенная по экспериментальным кривым ползучести математическая модель ползучести разупрочняющегося материала на примере алюминиевого сплава Д16Т при температуре $250^{\circ} \mathrm{C}$ имеет вид

$$
\hat{t}_{k, j}=\frac{1}{1.2543 \cdot 10^{-13} \sigma_{0 j}^{5.449}}\left(1-\exp \left(-0,861 \sigma_{0 j} p_{k, j}\right)\right) .
$$


Исходя из этого в качестве зависимости деформации ползучести от времени будем использовать функцию вида

$$
p\left(t, \sigma_{0 j}\right)=-\frac{1}{0.861 \sigma_{0 j}} \ln \left(1-2.254 \cdot 10^{-13} \sigma_{0 j}^{5.449} t\right) .
$$

Остаточные суммы квадратов для каждой $j$-той кривой ползучести в относительных единицах составили: 24.2, 8.8, 7.8, 11.3 и $4.9 \%$ соответственно. По совокупности всех кривых остаточная сумма квадратов в относительных единицах равна $8.3 \%$. Коэффициент детерминации $R^{2}$, который косвенно может служить оценкой адекватности построенной модели экспериментальным данным по совокупности всех кривых ползучести, составляет $R^{2}=0.96$, что свидетельствует о высокой степени адекватности построенной модели (19) данным эксперимента [11].

На основе статистического анализа результатов математического моделирования найдены оценки погрешности для параметров модели и построены доверительные интервалы для кривых ползучести. На рисунке при различных номинальных напряжениях $\sigma_{0 j}$ изображены:

- экспериментальные кривые (точки);

- кривые (сплошные линии), построенные на основе разработанной модели (19);

- границы доверительных интервалов (штриховые линии), вычисленные с использованием неравенства Чебышева при доверительной вероятности $\beta=0.97$.

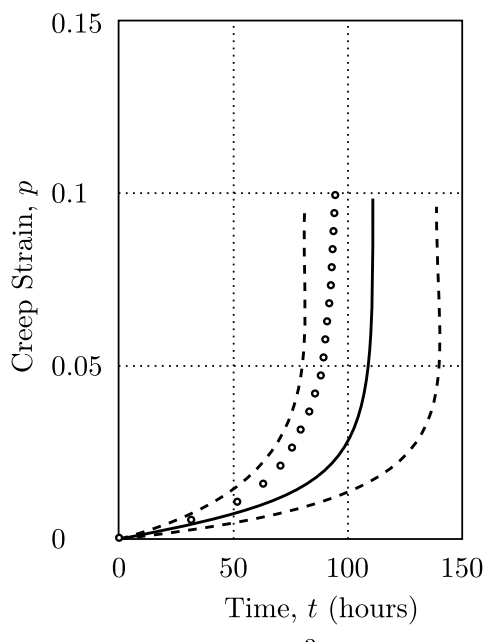

a
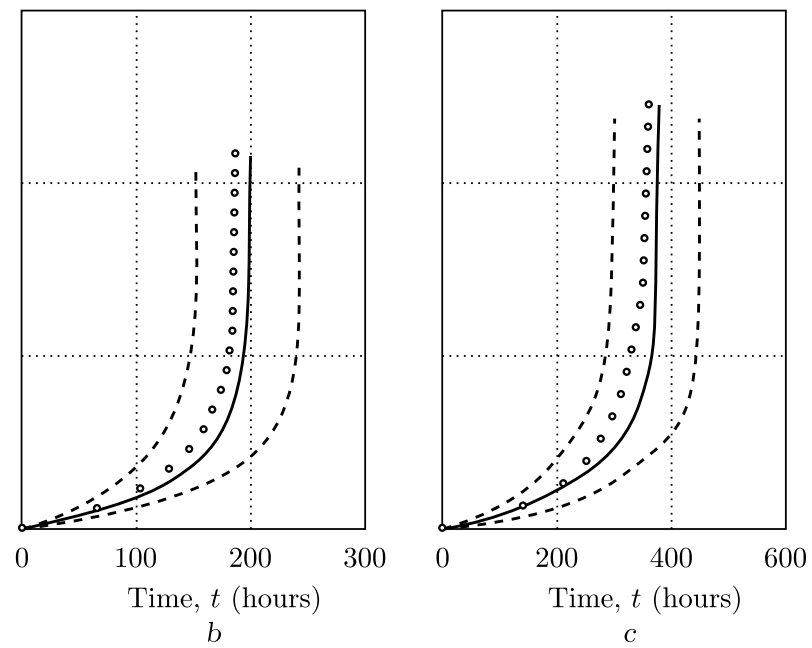

Расчетные доверительные интервалы на основе построенной модели (19) при различных значениях номинального напряжения: $\sigma_{01}=98.1 \mathrm{MПа}(\mathrm{a}), \sigma_{02}=83.3 \mathrm{MПа}(b)$, $\sigma_{03}=78.5 \mathrm{M \Pi а}(c)$ [Calculated confidence intervals on the basis of the constructed model (19) at different values of the nominal stress: $\sigma_{01}=98.1 \mathrm{MPa}(\mathrm{a}), \sigma_{02}=83.3 \mathrm{MPa}(\mathrm{b})$, $\left.\sigma_{03}=78.5 \mathrm{MPa}(c)\right]$

Выводы. Таким образом, разработан новый численный метод оценивания параметров модели ползучести, описывающей разупрочнение на третьей стадии, по совокупности кривых ползучести, построенных при обработке результатов эксперимента для различных значений номинального напряжения. 
В основе метода лежат разностные уравнения, описывающие результаты эксперимента, сформированная на их базе обобщенная регрессионная модель, коэффициенты которой известным образом связаны с параметрами модели ползучести, и итерационная процедура среднеквадратического оценивания коэффициентов обобщенной регрессионной модели. Такой подход к решению задачи нелинейного оценивания позволяет минимизировать величину отклонения модели от данных эксперимента и тем самым обеспечить высокую точность оценок параметров модели. Результаты апробации нового численного метода при обработке результатов эксперимента в форме кривых ползучести алюминиевого сплава Д16Т при температуре $250{ }^{\circ} \mathrm{C}$ подтвердили достоверность полученных соотношений и выводов, а также высокую эффективность численного метода в задачах оценивания параметров рассматриваемой модели ползучести.

\section{ORCIDs}

Владимир Евгеньевич Зотеев: http://orcid.org/0000-0001-7114-4894

Роман Юрьевич Макаров: http://orcid.org/0000-0001-8620-1222

\section{БИБЛИОГРАФИЧЕСКИЙ СПИСОК}

1. Малинин Н. Н. Прикладная теория пластичности и ползучести. М.: Машиностроение, 1975. 387 с.

2. Соснин О. В., Любашевская И. В., Новоселя И. В. Сравнительные оценки высокотемпературной ползучести и разрушения конструкционных материалов // ПМТФ, 2008. T. 49, № 2. C. 123-130.

3. Bellenger E., Bussy P. Phenomenological modeling and numerical simulation of different modes of creep damage evolution // International Journal of Solids and Structures, 2001. vol. 38, no. 4. pp. 577-604. doi : 10.1016/S0020-7683(00)00042-1.

4. Расчеты и испытания на прочность. Расчетные методы определения несущей способности и долговечности элементов машин и конструкиий. М.: ВНИИМАШ, 1982. $90 \mathrm{c.}$

5. Радченко В. П., Еремин Ю. А. Реологическое деформирование и разрушения материалов и элементов конструкиий. М.: Машиностроение-1, 2004. 264 с.

6. Демиденко Е. З. Линейная и нелинейная регрессия. М.: Финансы и статистика, 1981. $302 \mathrm{c}$.

7. Зотеев В. Е. Параметрическая идентификация диссипативных механических систем на основе разностных уравнений/ ред. В. П. Радченко. М.: Машиностроение, 2009. $344 \mathrm{c}$.

8. Зотеев В. Е., Заусаева М. А. Определение параметров двумерных динамических процессов на основе разностных схем // Вестн. Сам. гос. техн. ун-та. Сер. Физ.-мат. науки, 2010. № 1(20). С. 154-161. doi : 10.14498/vsgtu781.

9. Зотеев В. Е. Математические основы построения разностных уравнений для задач параметрической идентификации // Вестн. Сам. гос. техн. ун-та. Сер. Физ.-мат. наукu, 2008. № 2(17). С. 192-202. doi : 10.14498/vsgtu623.

10. Зотеев В. Е. Параметрическая идентификация линейной динамической системы на основе стохастических разностных уравнений // Матем. моделирование, 2008. Т. 20, № 9. С. 120-128.

11. Соснин О. В., Горев Б. В., Никитенко А. Ф. Энергетический вариант теории ползучести. Новосибирск: ИГиЛ СО АН СССР, 1986. 95 с.

Поступила в редакцию $21 / \mathrm{III} / 2016$;

в окончательном варианте - 13/V/2016;

принята в печать $-27 / \mathrm{V} / 2016$. 
Vestn. Samar. Gos. Techn. Un-ta. Ser. Fiz.-mat. nauki

[J. Samara State Tech. Univ., Ser. Phys. \& Math. Sci.], 2016, vol. 20, no. 2, pp. 328-341

ISSN: 2310-7081 (online), 1991-8615 (print)

doi: http://dx.doi.org/10.14498/vsgtu1488

MSC: $74 S 30$

\title{
A NUMERICAL METHOD FOR THE DETERMINATION OF PARAMETERS OF THE STRAIN SOFTENING CREEP MODEL
}

\section{E. Zoteev, R. Yu. Makarov}

Samara State Technical University,

244, Molodogvardeyskaya st., Samara, 443100, Russian Federation.

\begin{abstract}
The trends of decreasing of weight of machines and increasing of their quality, and intention to the fullest use of mechanic properties of materials demand the development of numerical methods for analysis of the stress-strain state of materials undo the terms of creep. The article discusses the development of new numerical method for determining the parameters of the strain softening creep model. The base of new method is generalized regression model, which was built on the basis of difference equations for describing the creep. The relations between coefficients of difference equation and parameters of the strain softening creep model allow reduce the problem of parametric identification to an iterative procedure for RMS of coefficients of regression model, which is linear. The approbation of numerical method with five creep curves of aluminum alloy is accomplished. The approbation confirms scientific credibility of built relations and efficiency of new numerical method.
\end{abstract}

Keywords: strain softening creep model, difference equations, generalized regression model, nonlinear regression, root mean square evaluation.

\section{ORCIDs}

Vladimir E. Zoteev: http://orcid.org/0000-0001-7114-4894

Roman Yu. Makarov: http://orcid.org/0000-0001-8620-1222

\section{REFERENCES}

1. Malinin N. N. Prikladnaia teoriia plastichnosti i polzuchesti [Applied theory of plasticity and creep]. Moscow, Mashinostroenie, 1975, 387 pp. (In Russian)

2. Sosnin O. V., Lyubashevskaya I. V., Novoselya I. V. Comparative estimation of hightemperature creep and rupture of structural materials, J. Appl. Mech. Tech. Phys., 2008, vol. 49, no. 2, pp. 262-266. doi: 10.1007/s10808-008-0036-0.

(C) 2016 Samara State Technical University.

Please cite this article in press as:

Zoteev V. E., Makarov R. Yu. A numerical method for the determination of parameters of the strain softening creep model, Vestn. Samar. Gos. Tekhn. Univ., Ser. Fiz.-Mat. Nauki [J. Samara State Tech. Univ., Ser. Phys. \& Math. Sci.], 2016, vol. 20, no. 2, pp. 328-341. doi: 10.14498/vsgtu1488. (In Russian)

\section{Authors Details:}

Vladimir E. Zoteev (Dr. Techn. Sci.; zoteev-ve@mail.ru; Corresponding Author), Professor, Dept. of Applied Mathematics \& Computer Science.

Roman Yu. Makarov (makaroman1@yandex.ru), Postgraduate Student, Dept. of Applied Mathematics \& Computer Science. 
3. Bellenger E., Bussy P. Phenomenological modeling and numerical simulation of different modes of creep damage evolution, International Journal of Solids and Structures, 2001, vol. 38, no. 4, pp. 577-604. doi: 10.1016/S0020-7683(00)00042-1.

4. Raschety $i$ ispytaniia na prochnost'. Raschetnye metody opredeleniia nesushchei sposobnosti $i$ dolgovechnosti elementov mashin $i$ konstruktsii [Design calculations and strength testing. Calculation methods for the determination of bearing capacity and durability of machine elements and constructions]. Moscow, All-Russian scientific research institute for standardization and certification in the branch of mechanical engineering, 1982, 90 pp. (In Russian)

5. Radchenko V. P., Eremin Yu. A. Reologicheskoe deformirovanie i razrusheniia materialov $i$ elementov konstruktsii [Rheological deformation and fracture of materials and structural elements]. Moscow, Mashinostroenie-1, 2004, 264 pp. (In Russian)

6. Demidenko E. Z. Lineinaia $i$ nelineinaia regressiia [Linear and Nonlinear Regression]. Moscow, Finansy i statistika, 1981, 302 pp. (In Russian)

7. Zoteev V. E. Parametricheskaia identifikatsiia dissipativnykh mekhanicheskikh sistem na osnove raznostnykh uravnenii [Parametric Identification of Dissipative Mechanical Systems Based on Difference Equation], ed. V. P. Radchenko. Moscow, Mashinostroenie, 2009, 344 pp. (In Russian)

8. Zausaeva M. A., Zoteev V. E. Definition of Parameters of 2D Dynamical Processes on the Basis of Difference Schemes, Vestn. Samar. Gos. Tekhn. Univ. Ser. Fiz.-Mat. Nauki [J. Samara State Tech. Univ., Ser. Phys. \& Math. Sci.], 2010, no.1(20), pp. 154-161 (In Russian). doi : 10.14498/vsgtu781.

9. Zoteev V. E. Mathematical base for difference equations formulation in parametrical identification problems, Vestn. Samar. Gos. Tekhn. Univ. Ser. Fiz.-Mat. Nauki [J. Samara State Tech. Univ., Ser. Phys. \& Math. Sci.], 2008, no. 2(17), pp. 192-202 (In Russian). doi: 10.14498/vsgtu623.

10. Zoteev V. E. Parametrical identification of linear dynamical system on the basis of stochastic difference equations, Matem. Mod., 2008, vol. 20, no. 9, pp. 120-128 (In Russian).

11. Sosnin O. V., Gorev B. V., Nikitenko A. F. Energeticheskii variant teorii polzuchesti [Energetic variant of the creep theory]. Novosibirsk, Institut Gidrodinamiki, 1986, 95 pp. (In Russian)

Received 21/III/2016;

received in revised form $13 / \mathrm{V} / 2016$;

accepted $27 / \mathrm{V} / 2016$. 